\title{
Biological and Clinicopathological Implications of Beta-3-N-acetylglucosaminyltransferase 8 in Triple-negative Breast Cancer
}

\author{
MISATO OKAZAKI ${ }^{1}$, KAORU MOGUSHI ${ }^{2}$, KAORI DENDA-NAGAI $^{3}$, HARUHIKO FUJIHIRA $^{3,4}$, \\ MIKI NOJI $^{3}$, KATRIN ISHII-SCHRADE ${ }^{3}$, MADOKA SAKATA-MATSUZAWA ${ }^{1}$, \\ KATSUYA NAKAI ${ }^{1}$, YOSHIYA HORIMOTO ${ }^{1,5}$, MITSUE SAITO $^{1}$ and TATSURO IRIMURA ${ }^{3}$ \\ ${ }^{1}$ Department of Breast Oncology, Juntendo University School of Medicine, Tokyo, Japan; \\ ${ }^{2}$ Intractable Disease Research Center, Juntendo University Graduate School of Medicine, Tokyo, Japan; \\ ${ }^{3}$ Division of Glycobiologics, Intractable Disease Research Center, \\ Juntendo University Graduate School of Medicine, Tokyo, Japan; \\ ${ }^{4}$ Glycometabolic Biochemistry Laboratory, Cluster for Pioneering Research, RIKEN, Saitama, Japan; \\ ${ }^{5}$ Department of Human Pathology, Juntendo University School of Medicine, Tokyo, Japan
}

\begin{abstract}
Background/Aim: Triple-negative breast cancer (TNBC) remains difficult to treat and new molecular targets are needed. Here, we investigated the impact of glycosyltransferase genes on TNBC patient survival. Patients and Methods: mRNA expression levels of 101 glycosyltransferase genes in TNBC patients were compared for correlation with patient survival using The Cancer Genome Atlas data. An antibody to $\beta-3-N-$ acetylgluco-saminyltransferase 8 (B3GNT8) was applied to investigate B3GNT8 protein distribution and expression levels in 23 TNBC surgical specimens. Results: B3GNT8 mRNA levels inversely correlated with relapse-free survival $(p<0.01)$ and overall survival $(p<0.05)$ in TNBC patients. Anti-B3GNT8 antibody binding was observed as dots in the cytoplasm of cancer cells. These dots were supposed to correspond to B3GNT8 protein in tumour cells, but their number was smaller in relapsed patients than in non-relapsed patients. Conclusion: B3GNT8 mRNA expression levels in TNBC tumour tissues are potentially useful in distinguishing patients with favourable and poor clinical outcomes.
\end{abstract}

This article is freely accessible online.

Correspondence to: Tatsuro Irimura, Ph.D., and Kaori DendaNagai, PhD, Division of Glycobiologics, Intractable Disease Research Center, Graduate School of Medicine, Juntendo University, 2-1-1 Hongo, Bunkyo-ku, Tokyo 113-8421, Tokyo, Japan. Tel: +81358021876 (TI) or +81338133111 (KD-N), Fax: +81338308715, e-mail: t-irimura@juntendo.ac.jp (TI), kdenda@juntendo.ac.jp (KD-N)

Key Words: $\beta-3-N$-acetylglucosaminyltransferase 8 , prognostic factor, triple-negative breast cancer.
Triple-negative breast cancer (TNBC) comprises approximately $15 \%$ of breast cancers and is defined by the lack of oestrogen receptor (ER), progesterone receptor $(\mathrm{PgR})$, and human epidermal growth factor receptor-2 (HER2) overexpression $(1,2)$. Because of the lack of therapeutic molecular targets, TNBC patients receive standard chemotherapy treatments, commonly anthracycline followed by taxane. However, in the adjuvant setting only about one third of TNBC patients respond to such therapy. In patients who still have residual cancer after these treatments, the prognosis is poor (3). We focused on cellular glycosylation, because it has been shown by many experimental and clinical investigations that it is involved in malignant progression and metastasis in cancer $(4,5)$. In breast cancer, glycosylation has been implicated in cancer cell growth and metastasis (6). Song and co-workers showed that GALNT14, a glycosyltransferase, which transfers $N$ acetylgalactosamine (GalNAc) to polypeptides, promotes lung-specific breast cancer metastasis (7). As an upstream event, a transcriptional activator protein, LAMTOR5, was reported to modulate GALNT1 activity in breast cancer leading to abnormal $O$-glycosylation (8). However, the biological and clinical significance of glycosylation in TNBC has not been previously investigated.

Since glycosylation is controlled by glycosyltransferases, we conducted a comprehensive search on 101 glycosyltransferase genes in TNBC patients who did not receive neoadjuvant chemotherapy for correlation with their survival using The Cancer Genome Atlas (TCGA) mRNA expression database. From this search, $\beta-3-N$-acetylglucosaminyltransferase 8 (B3GNT8) emerged as a gene whose expression inversely correlated with the survival of TNBC patients. B3GNT8 is a 
member of the B3GNT family and it transfers $N$ acetylglucosamine (GlcNAc) to galactose residues of the nonreducing terminus of poly- $N$-acetyllactosamine structures (911). Poly- $N$-acetyllactosamine comprises a repeated $-3 \mathrm{Gal} \beta 1$ 4GlcNAc $\beta 1$ - and attaches to $O$-glycans, $N$-glycans, or glycolipids.

Poly- $N$-acetyllactosamine has long been implicated in cancer progression and metastasis (12). Ishida and coworkers recently showed that B3GNT8 expression is dramatically upregulated in colon carcinoma cells and provided evidence that this enzyme is involved in the synthesis of poly- $N$-acetyllactosamine on $\beta 1-6$ branched $N$ glycans in colon carcinoma cells (11). In another study, $\mathrm{Ni}$ and co-workers showed that BG3NT8 regulates the metastatic potential of colorectal carcinoma cells by altering the matrix metalloprotease-inducing function of CD147 (13). Jiang and co-workers showed that B3GNT8 overexpression in colon carcinoma cells promoted the invasion of cells through changes in glycosylation of CD147 driven by B3GNT2, whereas B3GNT8 knockdown inhibited the invasion (14). Furthermore, knockdown of B3GNT8 in human colorectal carcinoma cell lines reverted chemotherapeutic drug resistance by suppressing the formation of poly- $\mathrm{N}$-acetyllactosamine, according to Shen and co-workers (15). Despite these extensive investigations on the involvement of B3GNT8 in colon carcinoma biology, the role of this enzyme in the malignant behaviour of TNBC has not been reported. Therefore, it is intriguing that $B 3 G N T 8$ mRNA levels were found to be correlated with poor survival in TNBC patients. Thus, B3GNT8 mRNA expression potentially serves as a clinically useful indicator of patient survival independent of other clinicopathological variables. In the present study, B3GNT8 protein expression and distribution were investigated in surgical specimens from TNBC patients by antibody binding. The subcellular localization was unique. The binding sites were distributed as a small number of dot-like structures. The possible molecular and cellular basis of these findings is discussed.

\section{Patients and Methods}

Comparisons of the expression levels of glycosyltransferase genes in TNBC between relapsed and non-relapsed patients. The Breast Invasive Carcinoma RNA-seq data set of 817 breast cancer patients from the cBioPortal Cancer Genomics website (16) was downloaded. TCGA cancers selected for this study were originally published by the National Cancer Institute (17). Intrinsic classification results by PAM50 were obtained by the "TCGAbiolinks" package using the $\mathrm{R}$ project for statistical computing (18). TNBC patients were selected according to the following criteria: ER (-) by immunohistochemistry (IHC), PgR (-) by IHC, and HER 2 score 0 or 1 by IHC. Further, among patients with a HER2 score of 2 by IHC, those with HER2 fluorescence in situ hybridization (FISH) negative result were selected. In addition, for patients with a HER2 score of 2 by IHC and no available FISH data, those with PAM50 intrinsic classification as triple-negative (19) were selected. Finally, patients with a HER2 score of 2 by IHC with neither FISH data nor PAM50 data were excluded from analysis.

Relapse-free survival (RFS) and overall survival (OS) analyses were performed using Graphpad Prism 8 (GraphPad Software, San Diego, CA, USA). Patients were divided into higher- and lowerexpression groups, using the median of the B3GNT8 mRNA expression level ( $\log 2$ values) as a cut-off. The relationship between $B 3 G N T 8$ mRNA expression and clinicopathological variables was analysed using GraphPad Prism 8 software, and statistical significance was evaluated by the Mann-Whitney $U$-test or the Kruskal-Wallis test where appropriate.

To investigate the relationship between B3GNT8 mRNA expression and clinicopathological variables, patients were stratified into Stages I to IV and classified into TNBC subtypes by applying Lehmann's TNBCtype-4 classification (20). Differences in B3GNT8 mRNA expression between patients with relapse and patients without relapse were analysed by the Mann-Whitney U test using a cut-off median. A $p$-value $<0.05$ was considered statistically significant.

Research involving human participants. The study was carried out with approval from the Ethics Committee of Juntendo University Hospital (no: 2017013) and has been performed in accordance with the ethical standards laid down in the 1964 Declaration of Helsinki and its later amendments or comparable ethical standards.

Informed consent. All specimens for immunohistochemistry were collected after patient consent, which was obtained by opt-out using the home page provided by Juntendo University's ethical committee.

TNBC cell lines. MDA-MB-468, HCC1937, HCC70, DU4475, HCC1187, HCC2157, HCC1806 and BT549 cells were cultivated in RPMI1640 (Thermo Fisher Scientific, Waltham, MA, USA). Hs578T cells were cultured in DMEM (Thermo Fisher Scientific). MDA-MB-453, MDA-MB-231, BT20, and MRK-nu-1 cells were cultured in DMEM/F-12 (Thermo Fisher Scientific). Medium supplements were added as specified in the American Type Culture Collection product sheet for each cell line, except for MDA-MB231, MDA-MB-453, and BT-20, which were cultured in DMEM/F12 supplemented with $10 \%$ FCS and MDA-MB-468, which was cultured in RPMI 1640 supplemented with HEPES and 10\% FCS. All cell lines were cultured in a humidified atmosphere with $5 \%$ $\mathrm{CO}_{2}$ at $37^{\circ} \mathrm{C}$.

Quantitative real-time PCR analysis. Total RNA of TNBC cell lines was isolated using PureLink RNA mini kit (Thermo Fisher Scientific) according to the manufacturer's protocol. Five micrograms of isolated total RNA were reverse transcribed with SuperScript IV reverse transcriptase (Thermo Fisher Scientific). One of twenty-two equal parts of the resulting cDNA was used for quantitative real-time PCR analysis using SYBR Green Master Mix Kit and a 7500 Fast Realtime PCR System (Applied Biosystems, Foster City, CA, USA). The following PCR primers were used: $A C T B$ forward; 5'-CAGACGC TCAAAGACCTGCT-3' and reverse; 5'-AGCTCGCAAGACAAAA CTCAC-3', B3GNT8 forward; 5'-CAGACGCTCAAAGACCTGCT3 ' and reverse 5'-AGCTCGCAAGACAAAACTCAC-3'. The relative expression data were calculated by $2^{-\Delta \Delta \mathrm{Ct}}$ method using $A C T B$ mRNA expression as internal control. 
Construction of B3GNT8-expressing plasmid, plasmid transfection and establishment of B3GNT8-overexpressing cells. The B3GNT8encoding plasmid pcDNA3.1-B3GNT8-FLAG was kindly provided by Dr. Kiyohiko Angata (National Institute of Advanced Industrial Science and Technology, Tokyo, Japan). The IRES-Venus sequence was amplified by PCR using specific primers (forward primer: 5'CGCGCGGCCGCTTCGCGGATCCGCCCCTCTCCC-3', reverse primer: 5'-CGCTCTAGAGGCTCGAGAGGCCT-3'), and CSII-EFMCS-IRES2-Venus plasmid (RIKEN Bio Resource Center, Tsukuba, Japan) was used as the template. Amplified IRES-Venus fragment and pcDNA3.1-B3GNT8-FLAG plasmid were digested with NotI and XbaI (New England Biolabs, Ipswich, MA, USA), and the digested IRES-Venus fragment was ligated to the B3GNT8FLAG sequence (pcDNA3.1-B3GNT8-FLAG-IRES-Venus). The sequence of the constructed plasmid was confirmed by Direct DNA sequencing (FASMAC, Kanagawa, Japan). BT20 cells $\left(5 \times 10^{5}\right.$ cells $)$ were seeded on a 6-well plate one day before transfection. Cells were transfected with pcDNA3.1-B3GNT8-FLAG-IRES-Venus by using Lipofectamine 3000 (Thermo Fisher SCIENTIFIC, Waltham, MA, USA) according to the manufacturer's protocol. Forty-eight hours after transfection, cells were washed with PBS and subsequently cultured in new medium containing $200 \mu \mathrm{g} / \mathrm{ml}$ of G418. Eleven days after G418 selection, Venus expression was confirmed and high-expressing cells were enriched by sorting with a BD FACSAria III (Becton Dickinson, Franklin Lakes, NJ, USA). The enriched cells were used as B3GNT8-overexpressing cells.

Confirmation of B3GNT8-overexpressing cells by SDS-PAGE and western blotting analysis. Cultured cells were washed with PBS and harvested using a cell scraper. Cells were lysed with lysis buffer (10 mM Tris-HCl pH 7.2, $0.25 \mathrm{M}$ sucrose, $0.05 \mu \mathrm{M} \mathrm{CaCl}_{2}$, $0.5 \%$ NP-40, $1 / 200$ diluted protease inhibitor cocktail III). Protein concentration was measured with the BCA assay (Thermo Fisher Scientific). Twenty micrograms of protein lysates were loaded per lane, separated by SDS-PAGE, and blotted on a polyvinylidene difluoride membrane (Millipore, Burlington, MA, USA). The membrane was blocked with Bullet Blocking One (Nacalai Tesque, Kyoto, Japan) and incubated with anti-B3GNT8 antibody (NSJ Bioreagents, San Diego, CA, USA, $0.5 \mu \mathrm{g} / \mathrm{ml}$ ) at $4^{\circ} \mathrm{C}$ overnight followed by HRP-goat anti-rabbit antibody (Jackson Immuno Research Laboratories, West Grove, PA, USA, 1/3,000) and incubated for $45 \mathrm{~min}$ at room temperature. The band was visualised using Clarity Western ECL detection reagent (Bio Rad, Hercules, CA, USA).

Patient selection, immunocytochemistry and immunohistochemistry. During the period from May 2006 to September 2017, 112 TNBC patients underwent curative surgery at Juntendo University Hospital. We examined surgical specimens rather than biopsy specimens in order to reduce effects possibly introduced by tumour heterogeneity. In addition, we selected patients who had not received preoperative chemotherapy but postoperative standard chemotherapy regimens to minimise any bias stemming from differential tumour cytotoxicity effects. The flow of patient selection is shown in Figure 1. Based on the patient selection criteria, we included 23 Stage II cases whose surgical specimens were available into the current study. All patients received standard systemic chemotherapies following surgery.

Polyclonal rabbit anti-B3GNT8 antibody (Atlas, Bromma, Sweden) was used for immunocytochemical and immunohistochemical staining. Specificity of the antibody was confirmed using BT-20 cells

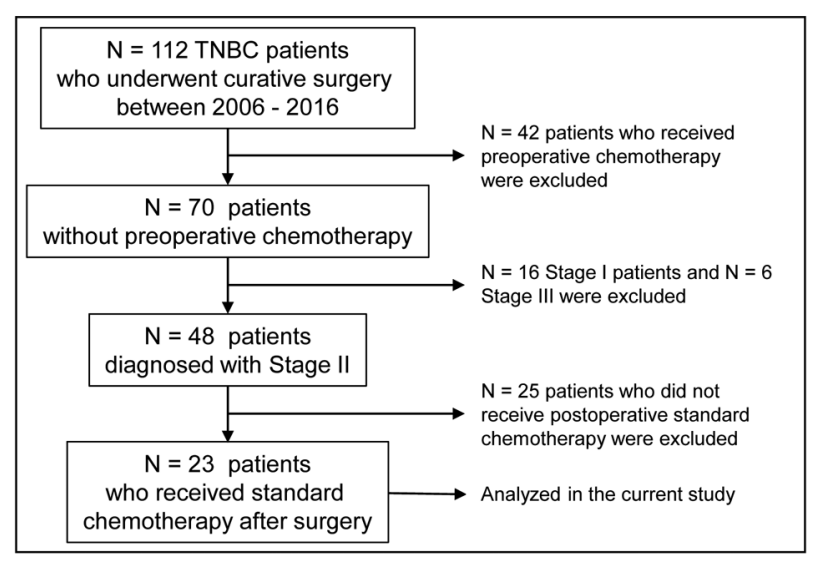

Figure 1. Patient selection flow chart.

transfected with B3GNT8-encoding plasmid pcDNA3.1-B3GNT8FLAG. Five thousand B3GNT8-overexpressing BT-20 cells and three thousand mock BT-20 cells were seeded on Lab-Tek II chamber slides (Nunc, Roskilde, Denmark) 4 days before staining. Cells were washed with PBS and fixed with PBS containing 4\% (w/v) paraformaldehyde at room temperature for $15 \mathrm{~min}$.

Immunohistochemical staining of breast cancer tissue was performed on $4-\mu \mathrm{m}$ sections of formalin-fixed and paraffinembedded surgical specimens. Paraffin sections were deparaffinised in EZ Prep (Ventana Medical Systems, Oro Valley, AZ, USA). Antigen retrieval was carried out with CC1 (Ventana Medical Systems) for $1 \mathrm{~h}$.

Cell and tissue staining were performed using the $\mathrm{iView}^{\mathrm{TM}} \mathrm{DAB}$ Detection Kit (Ventana Medical Systems) on an automated staining apparatus, BenchMark-GX (Ventana Medical Systems), according to the manufacturer's instructions. As primary antibodies, rabbit anti-human B3GNT8 antibody (Atlas) $(1 \mu \mathrm{g} / \mathrm{ml}$ for BT-20 mock and BT-20 transfected cells; $4 \mu \mathrm{g} / \mathrm{ml}$ for tissue sections) was incubated for $32 \mathrm{~min}$ at $37^{\circ} \mathrm{C}$, and anti-FLAG antibody (clone M2, SigmaAldrich, St. Louis, MO, USA) ( $5 \mu \mathrm{g} / \mathrm{ml}$ for BT-20 mock and BT-20 transfected cells) was incubated for $120 \mathrm{~min}$ at $37^{\circ} \mathrm{C}$. Stained specimens were examined at various magnifications with an Olympus CX-41 microscope (Olympus, Tokyo, Japan). Because anti-B3GNT8 antibody binding to cancer cells in the surgical sections was observed as small, dot-like structures, the number of dots was counted in enlarged microscopic photographs assuming that this number represents the amount of B3GNT8 protein. The number of dots per cell from all patients were compared. Quantitative analysis was carried out using GraphPad Prism 6 software. Statistical significance was evaluated by the MannWhitney $U$-test. $p<0.05$ was considered statistically significant.

\section{Results}

Characteristics of TNBC patients chosen from the TCGA breast cancer data set and relationship of B3GNT8 mRNA expression with RFS and OS. To investigate whether the expression levels of any glycosyltransferase gene correlate to the survival of TNBC patients, 817 breast cancer patients in 
Table I. Clinicopathological characteristics of 109 TNBC patients from the TCGA data set. Patients were selected according to the following criteria: oestrogen receptor-negative, progesterone receptor-negative, human epidermal growth factor receptor 2-not overexpressed.

\begin{tabular}{lc}
\hline & Number of patients $(\%)$ \\
\hline Age (years) & \\
$30-39$ & $6(5.5)$ \\
$40-49$ & $25(22.9)$ \\
$50-59$ & $41(37.6)$ \\
$60-69$ & $21(19.3)$ \\
70 or greater $^{2}$ & $16(14.7)$ \\
Menopausal status $_{\text {Pre-menopausal }^{\mathrm{a}}}$ & $28(25.6)$ \\
Post-menopausal $^{\mathrm{b}}$ & $72(66.1)$ \\
Indeterminate $_{\text {Unknown }}$ & $3(2.8)$ \\
Pathological stage & $6(5.5)$ \\
I & \\
IIA/B & $19(17.4)$ \\
IIIA/B/C & $69(63.3)$ \\
IV & $18(16.5)$ \\
Unknown & $1(0.9)$ \\
\hline
\end{tabular}

aDefined as $<12$ months since last menstrual period AND no prior bilateral ovariectomy AND not on estrogen replacement. befined as prior bilateral ovariectomy $\mathrm{OR}>12$ months since last menstrual period with no prior hysterectomy.

the TCGA data set were investigated. Based on the definition for TNBC stated in the Material and Methods, 112 patients were selected as having TNBC. B3GNT8 mRNA expression data were available for 109 out of these 112 patients. The median values $(\log 2)$ and the range $(\min , \max )$ of B3GNT8mRNA expression levels were 5.013, 1.877, and 9.081, respectively. Approximately $37.6 \%$ of the patients were 50-59 years old, $80.7 \%$ of the patients had Stage I or II breast cancer, and only $0.9 \%$ of the patients presented with Stage IV breast cancer. Additional patient characteristics are shown in Table I. Overall, this TNBC cohort corresponds well to the patient population usually encountered in the clinical setting.

Among 101 glycosyltransferase genes tested, mRNA expression levels of B3GNT8 $(\log 2)$, with a median value of 5.013 (range $=1.877-9.080$ ), showed a significant inverse correlation with RFS and OS in this cohort. Patients with RFS had a significantly lower B3GNT8 expression level than patients who experienced cancer relapse $(p=0.003)$ (Figure 2A). Similarly, patients who survived had a significantly lower B3GNT8 expression levels compared to patients who eventually died of the disease $(p=0.017)$ (Figure 2B). Further, Kaplan-Meier analysis of the TCGA dataset revealed that recurrence-free survival was significantly worse in patients with high B3GNT8 expression ( $p=0.008$ ) (Figure $2 \mathrm{C})$. However, overall survival was not significantly different $(p=0.078)$ (Figure 2D).
RFS results were verified with another database from DNA microarray analysis, the Kaplan-Meier-Plotter database, employing a data set of 161 TNBC patients (21, 22) (Figure 3). Such a consistent correlation in separate databases was not observed with the expression levels of any other glycosyltransferase gene tested.

Relationship between B3GNT8 mRNA expression and clinicopathological variables. Further analysis revealed that B3GNT8 mRNA expression was independent of patient age (Figure 4A), menopausal status (Figure 4B), and histological diagnosis (Figure 4C). A significant difference in B3GNT8 mRNA levels was found between Stage II and Stage III patients $(p<0.01)$, but not between Stage I and Stage II patients (Figure 4D), showing that B3GNT8 mRNA expression levels do not consistently correlate with pathological Stage. When B3GNT8 expression levels were compared after classifying patients according to Lehmann's TNBCtype-4 (20), $B 3 G N T 8$ levels were significantly lower in patients with BL1 type than in patients with BL2, M, or LAR type $(p=0.0074)$ (Figure 4E). These results indicate that B3GNT8 mRNA expression is significantly lower in BL1 subtype.

TNBC patients were stratified according to Stages I to IV and then investigated for correlations between B3GNT8 mRNA levels and patient survival. The results revealed that median B3GNT8 levels were lower in relapse-free patients, but the differences were statistically insignificant (Stage II: $p=0.38$, Stage III: $p=0.122$ ) (Figure 5A). Lower levels of $B 3 G N T 8$ were observed in patients who were alive than in patients who eventually died of the disease, but the differences were not significant (Stage II: $p=0.48$, Stage III: $p=0.055$ ) (Figure 5B). Taken together, at least among Stage II and Stage III patients, patients with a better clinical outcome tended to have a lower B3GNT8 mRNA expression levels, suggesting that the prognostic value of B3GNT8 mRNA expression levels is independent of pathological stage.

When TNBC patients were stratified according to Lehmann's TNBCtype-4, median B3GNT8 levels were lower in relapse-free patients than in patients who experienced relapse or progression in all Lehmann's TNBCtype-4 subtypes. The difference was statistically significant in patients with BL2 type but not with other types (BL1: $p=0.056$, BL2: $p=0.03$, LAR: $p=0.43, \mathrm{M}: p=0.52$ ) (Figure $5 \mathrm{C})$. Patients who survived had lower B3GNT8 expression levels than patients who eventually died of the disease, except for patients with LAR type, and the differences were not statistically significant (BL1: $p=0.093$, BL2: $p=0.076$, LAR: $p=0.64, \mathrm{M}: p=0.34$ ) (Figure 5D). The results of Figure $5 \mathrm{C}$ and $\mathrm{D}$ indicate that lower B3GNT8 mRNA expression levels tended to be associated with better clinical outcome in patients with BL1, BL2, and $\mathrm{M}$ type, suggesting that B3GNT8 mRNA expression levels have a prognostic value for these TNBCtype-4 types. 

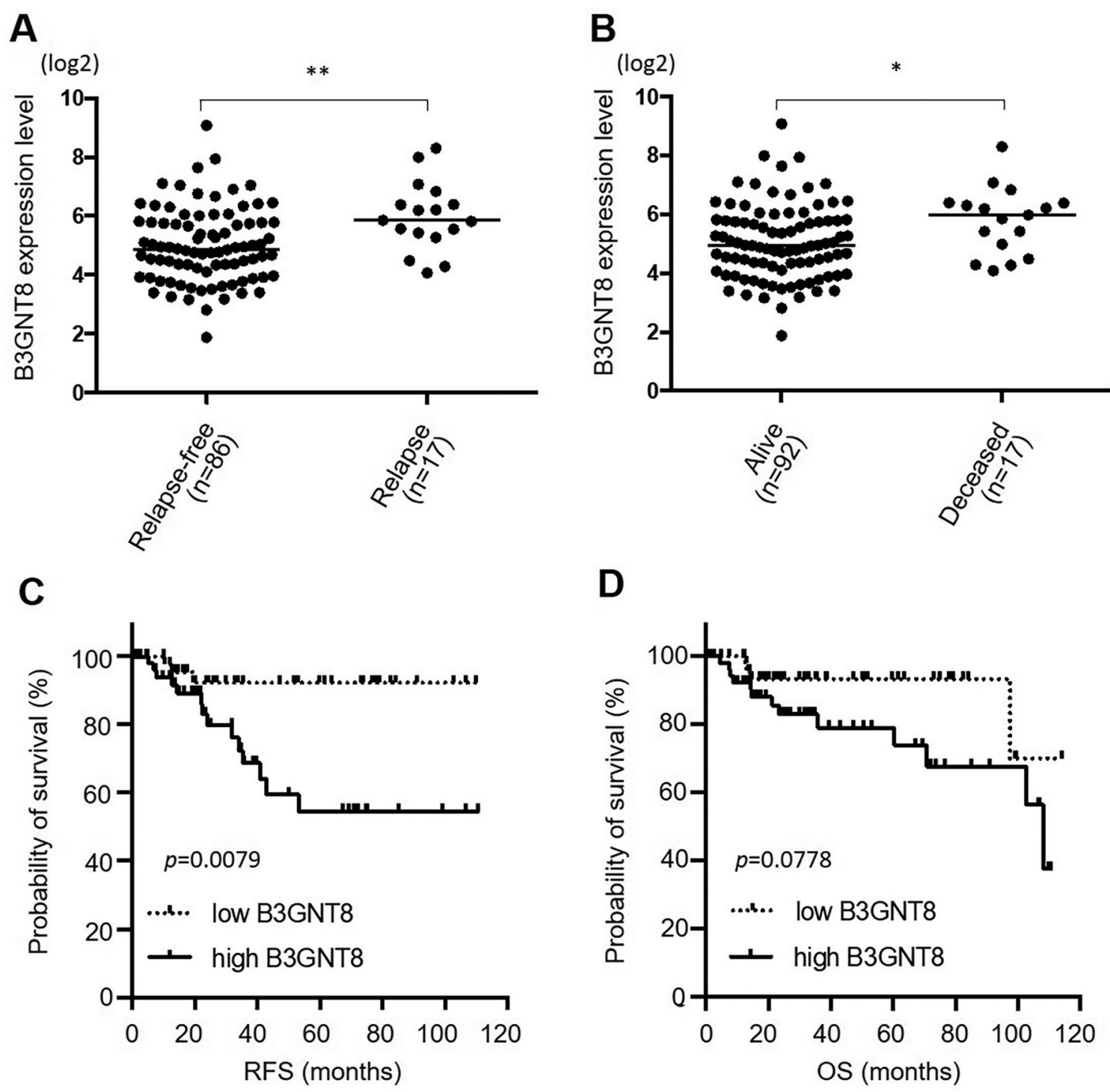

$\begin{array}{lllllll}\text { Number at risk } & & & & & \\ \text { Low } 51 & 29 & 18 & 14 & 7 & 4 & 0 \\ \text { High } 52 & 32 & 15 & 12 & 6 & 3 & 0\end{array}$

Number at risk

$\begin{array}{lllllll}\text { Low 53 } & 32 & 19 & 14 & 7 & 2 & 0 \\ \text { High 56 } & 35 & 20 & 15 & 9 & 7 & 0\end{array}$

Figure 2. B3GNT8 mRNA expression levels inversely correlate with relapse-free survival (RFS) and overall survival (OS) in triple-negative breast cancer (TNBC) patients in a TCGA dataset. An RNA-seq data set of 817 breast cancer patients was downloaded from the cBioPortal For Cancer Genomics website (16) and 112 patients were selected as having TNBC. Hundred and three and 109 patients were available for RFS and OS analysis, respectively. (A) B3GNT8 mRNA expression and RFS. (B) B3GNT8 mRNA expression and OS. p-values: two-tailed Mann-Whitney U-test. * $p<0.05$; ${ }^{* *} p<0.01$. The median of B3GNT8 expression level is shown as a line. (C) Kaplan-Meier survival curve, RFS. (D) Kaplan-Meier survival curve, OS. Patients were divided into a high and a low expression groups using the median of the B3GNT8 mRNA expression level (log2) as a cut-off (median: 5.013 , range $=1.877-9.080)$. p-values: log-rank test. The mean observation period was 35.8 months, the longest observation period was 110 months.

Immunohistochemical localisations of B3GNT8 protein in surgical specimens from TNBC patients. Thirteen TNBC cell lines were screened for B3GNT8 mRNA expression by realtime PCR (Figure 6A). To assess the binding and the specificity of anti-B3GNT8 polyclonal antibody, BT-20 cells were transfected with B3GNT8 plasmid vector. B3GNT8 mRNA overexpression in transfectants was confirmed by quantitative real-time PCR (Figure 6B). Protein expression was confirmed by western blotting analysis with a polyclonal anti-B3GNT8 antibody (Figure 6C). Staining of fixed cells 


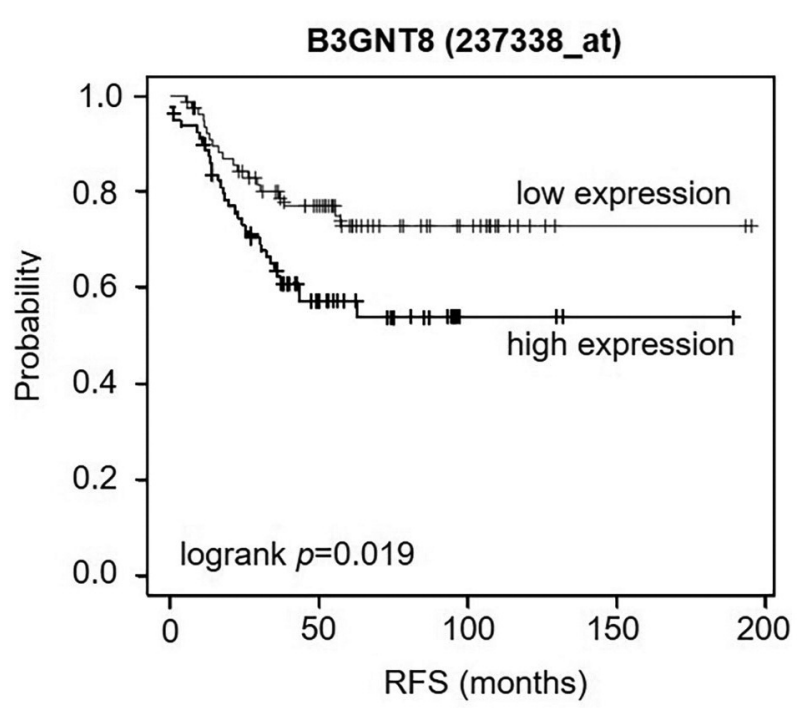

Number at risk

$\begin{array}{rrrrrr}\text { Low } & 81 & 46 & 17 & 2 & 0 \\ \text { High } & 80 & 28 & 3 & 1 & 0\end{array}$

Figure 3. High B3GNT8 mRNA expression correlates with shorter recurrence-free survival (RFS) in triple-negative breast cancer (TNBC) patients. The public database Kaplan-Meier Plotter (25) was used to search for RFS survival data in relation to B3GNT8 mRNA expression in the breast cancer data set with the Affymetrix ID 237338_at. TNBC patients were chosen by applying the following criteria: oestrogen receptor-negative, progesterone receptor-negative, and human epidermal growth factor receptor 2-negative. Patients were split at the median. A total of 161 TNBC patients were included in the analysis.

was performed with another anti-B3GNT8 polyclonal antibody alongside anti-FLAG antibody. Anti-B3GNT8 antibody binding was observed in the cytoplasm of BT-20 cells transfected with B3GNT8 (Figure 7A), while no staining was observed in BT-20 mock cells (Figure 7E) or controls (Figure 7B and F). Sites where strong binding was observed were the perinuclear area within the cytoplasm, likely to be associated with the Golgi apparatus. The staining pattern of anti-FLAG antibody was nearly identical to that of antiB3GNT8 antibody in B3GNT8-expressing cells, supporting the specificity of the anti-B3GNT8 antibody (Figure $7 \mathrm{C}, \mathrm{D}$, $\mathrm{G}$, and $\mathrm{H}$ ). These results indicate that this anti-B3GNT8 antibody can be used for immunohistochemistry of fixed, paraffin-embedded TNBC cells.

Anti-B3GNT8 antibody staining in sections of surgical TNBC specimens was observed as discrete dot-like structures within the cytoplasm of TNBC cells (Figure 7I and $\mathrm{K}$ ) compared to control (Figure $7 \mathrm{~J}$ and L).

Comparison of numbers of dot-like structures in relapse patients and non-relapse patients. Twenty-three surgical specimens from Stage IIA and IIB TNBC patients were investigated. Clinicopathological characteristics of these TNBC patients are shown in Table II. All patients had not been given neoadjuvant chemotherapy but had received postoperative chemotherapy uniformly according to the guideline of the Japanese Breast Cancer Society. Patient average age was 57.8 years (recurrence group 61.2 years, no recurrence group 54.8 years), and the average observation period was 52.1 months. B3GNT8 staining appeared as dots in the cytoplasm of cancer cells (Figure 7I and K). The number of dots varied among specimens. Some specimens had many and other specimens had few dots. Because dotlike structures were the only site where antibody-binding was observed, we assumed that the dot-like staining reflects the expression levels of B3GNT8 protein in these cells. One representative section of each TNBC patient was chosen and the numbers of dot-like structures in three randomly selected areas of $91,000 \mu \mathrm{m}^{2}$ were counted. The numbers of dots in tumour cells were visually counted on light microscopy photographs. The number of dots was normalised against the number of cancer cells present in the counting area. Figure 8 shows the comparisons of these values from 23 specimens plotted against their clinicopathological characteristics. As shown in Figure 8A, the number of dots was significantly greater in patients without disease-relapse $(p=0.01)$. Other parameters, such as age (Figure $8 \mathrm{~B}$ ), menopausal status (Figure 8C), pathological diagnosis (Figure 8D), and nuclear grade (Figure $8 \mathrm{E}$ ) did not show any correlation. Contrary to our hypothesis, the number of dots was significantly lower with tissue sections from patients having early relapse. Therefore, if the number of dots corresponds to the amount of B3GNT8 protein, the protein amount is high in TNBC cells in patients who did not have relapse.

\section{Discussion}

TNBC is a breast cancer subpopulation characterised by a lack of ER, PgR, and HER2 overexpression. Some, but not all TNBC patients experience rapid tumour growth, distant metastases, and early relapse after tumour resection. For this reason, molecular markers to identify subgroups having different clinical outcomes should provide important information for making therapeutic decisions and finding new therapeutic targets. In the present study, using a TCGA data set, we found that high levels of B3GNT8 mRNA in TNBC have an inverse correlation with RFS and OS. Such poor prognosis of patients with high B3GNT8 mRNA expression was confirmed with an additional independent database in the Kaplan-Meier Plotter (21), which includes gene expression data and survival information of 3951 breast cancer patients.

B3GNT8 is a glycosyltransferase involved in the extension of poly- $N$-acetyllactoseamine-type carbohydrate chains (11), and has been reported to play a key role in metastasis of 
A

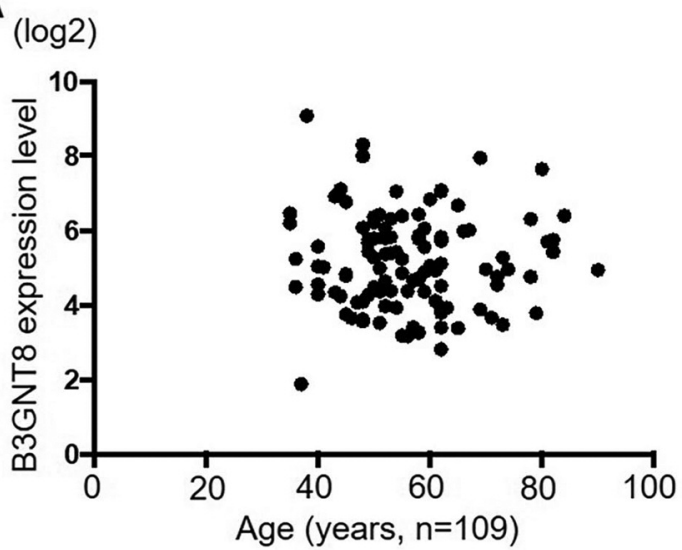

C

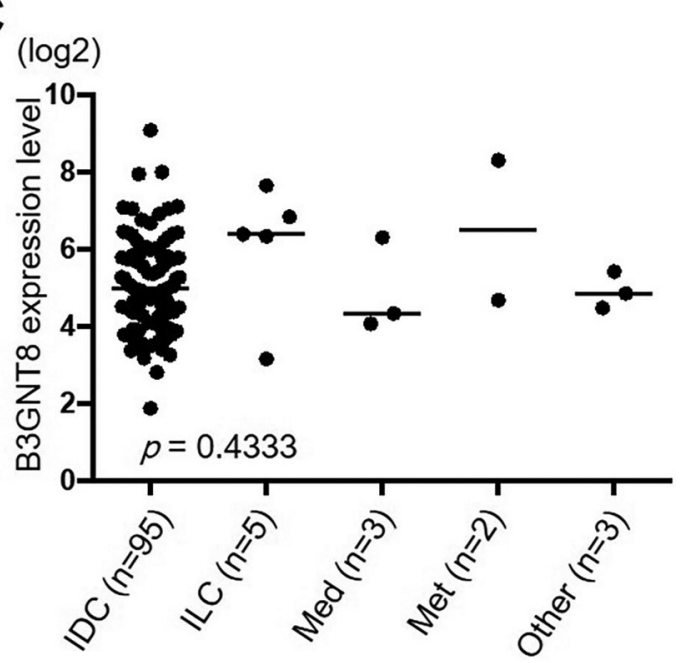

B

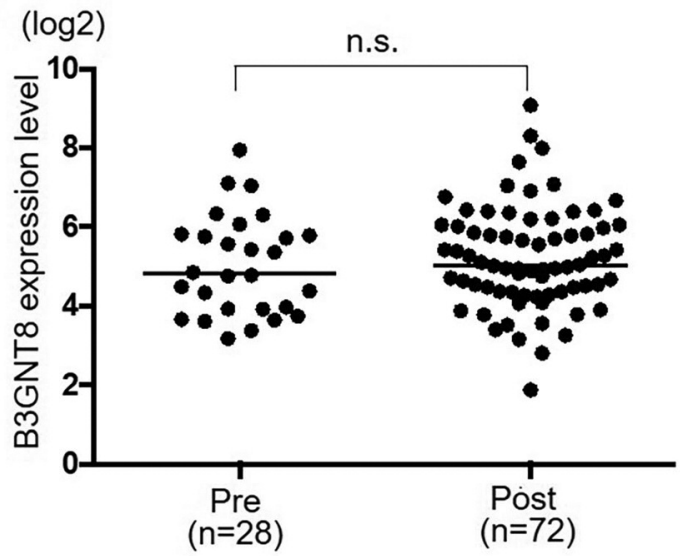

D

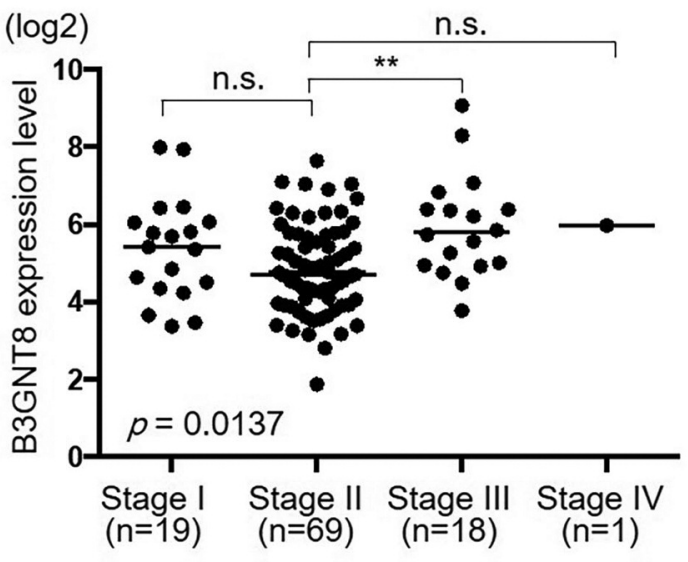

E

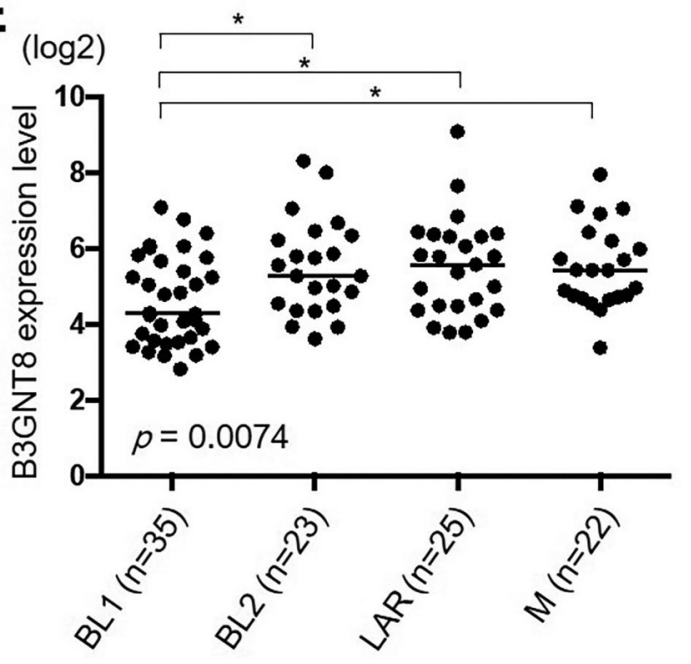

Figure 4. B3GNT8 mRNA expression and clinicopathological variables in 109 triple negative breast cancer (TNBC) patients in a TCGA dataset. (A) B3GNT8 mRNA expression and patient age. (B) B3GNT8 mRNA expression and menopausal status. Mann-Whitney U-test. n.s.; not significant. (C) B3GNT8 mRNA expression and breast cancer histological diagnosis. One specimen had no information about histological diagnosis and is not shown. IDC: invasive ductal carcinoma, ILC: invasive lobular carcinoma, Med: medullary carcinoma, Met: metaplastic carcinoma. Kruskal-Wallis test. (D) B3GNT8 mRNA expression and pathological TNM stage. Two patients had no information on cancer stage and are not shown. Kruskal-Wallistest (with Dunn's test as multiple comparison test). ${ }^{* *} p<0.01$, n.s. not significant. (E) B3GNT8 mRNA expression and Lehmann's TNBC-type4. Patients were classified into Lehmann's TNBCtype-4 (20). Four patients were not classifiable and are not shown. BL1; basal-like 1,BL-2; basallike 2, LAR; luminal androgen receptor, M; mesenchymal. KruskalWallis-test (with Dunn's test as multiple comparison test). ${ }^{*} p<0.05$. The median of B3GNT8 expression levels are shown as a horizontal line. 
A
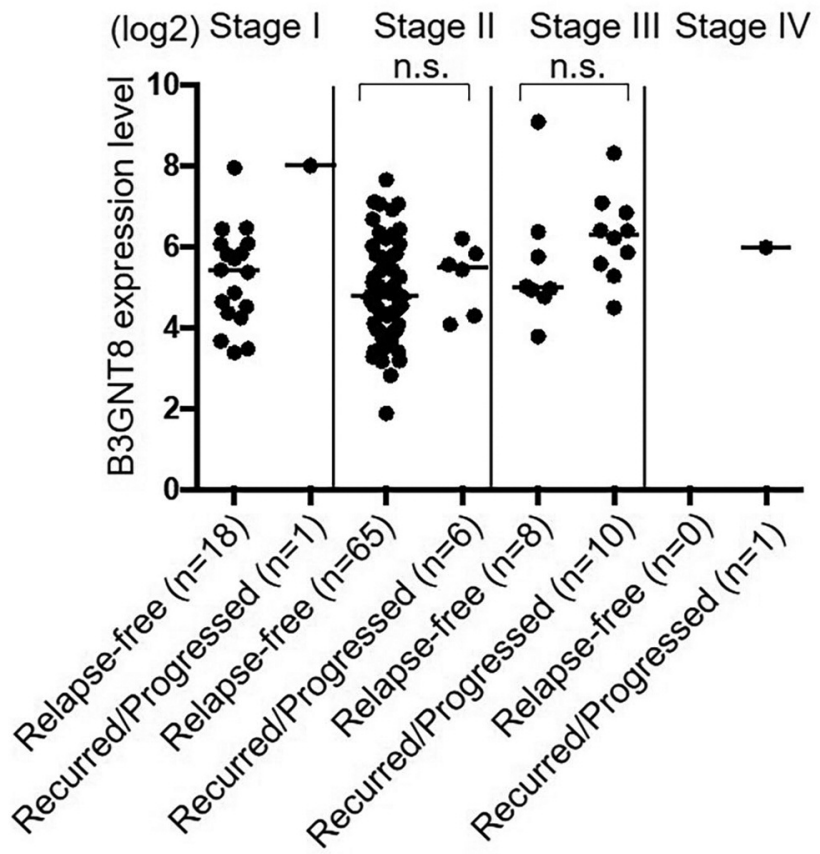

C

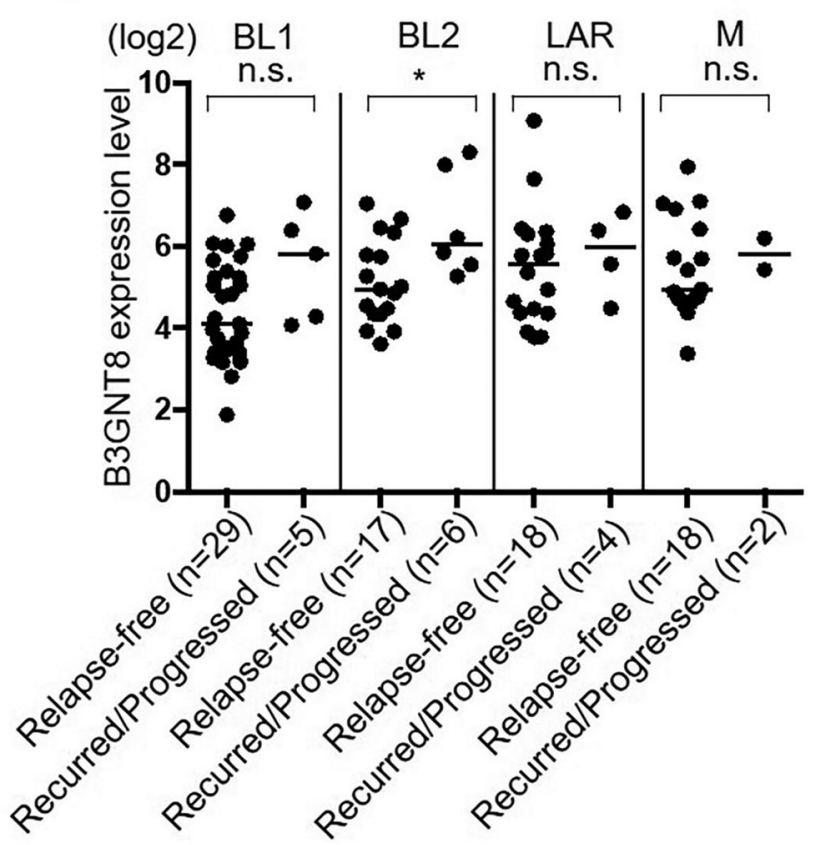

B

(log2) Stage I Stage II Stage III Stage IV
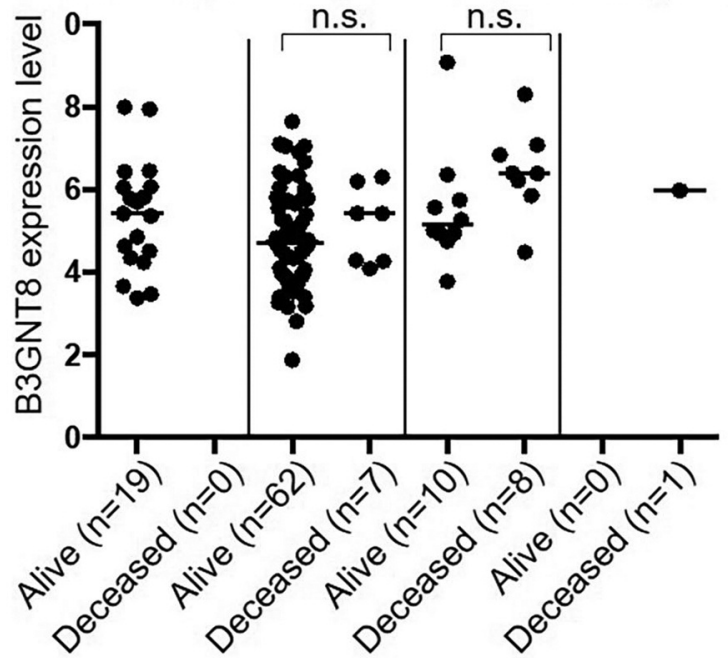

D

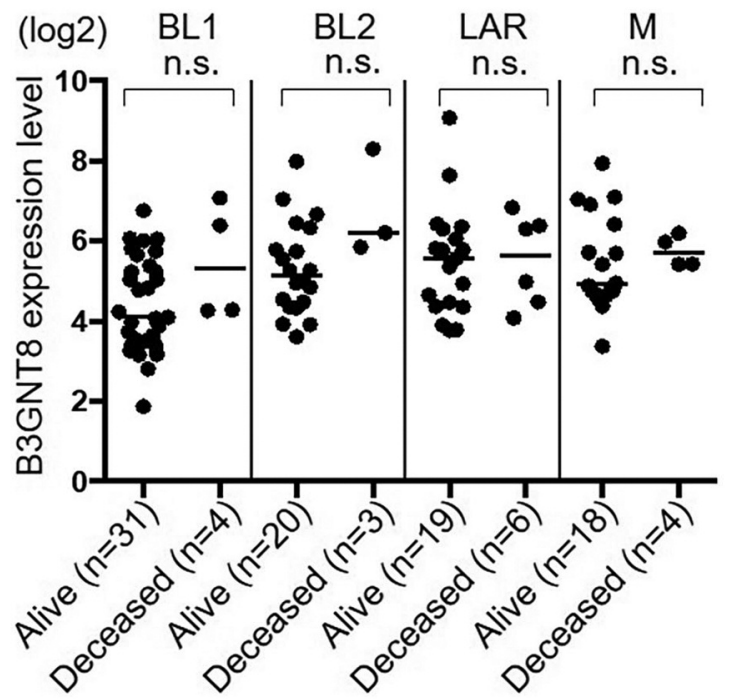

Figure 5. Correlation of B3GNT8 mRNA expression levels with disease relapse and overall survival in 109 triple negative breast cancer (TNBC) patients in a TCGA dataset. (A) B3GNT8 mRNA expression levels and disease relapse status in TNBC patients classified according to TMN stage. (B) B3GNT8 mRNA expression levels and overall survival in TNBC patients classified according to TMN stage. Two patients had no information on overall survival and are not shown. (C) B3GNT8 $\mathrm{mRNA}$ expression levels and disease relapse status in TNBC patients classified according to Lehmann's TNBCtype-4. Four patients who could not be classified into TNBC type and 6 patients who had no relapse information are not shown. (D) B3GNT8 mRNA expression levels and overall survival in TNBC patients classified according to Lehmann's TNBCtype-4. Four patients could not be classified into TNBC type and are not shown. BL1; basal-like 1, BL-2; basal-like 2, LAR; luminal androgen receptor, M; mesenchymal. Mann-Whitney U-test. n.s.; not significant, ${ }^{*} p<0.05$. The median of B3GNT8 expression is shown as a horizontal line. 


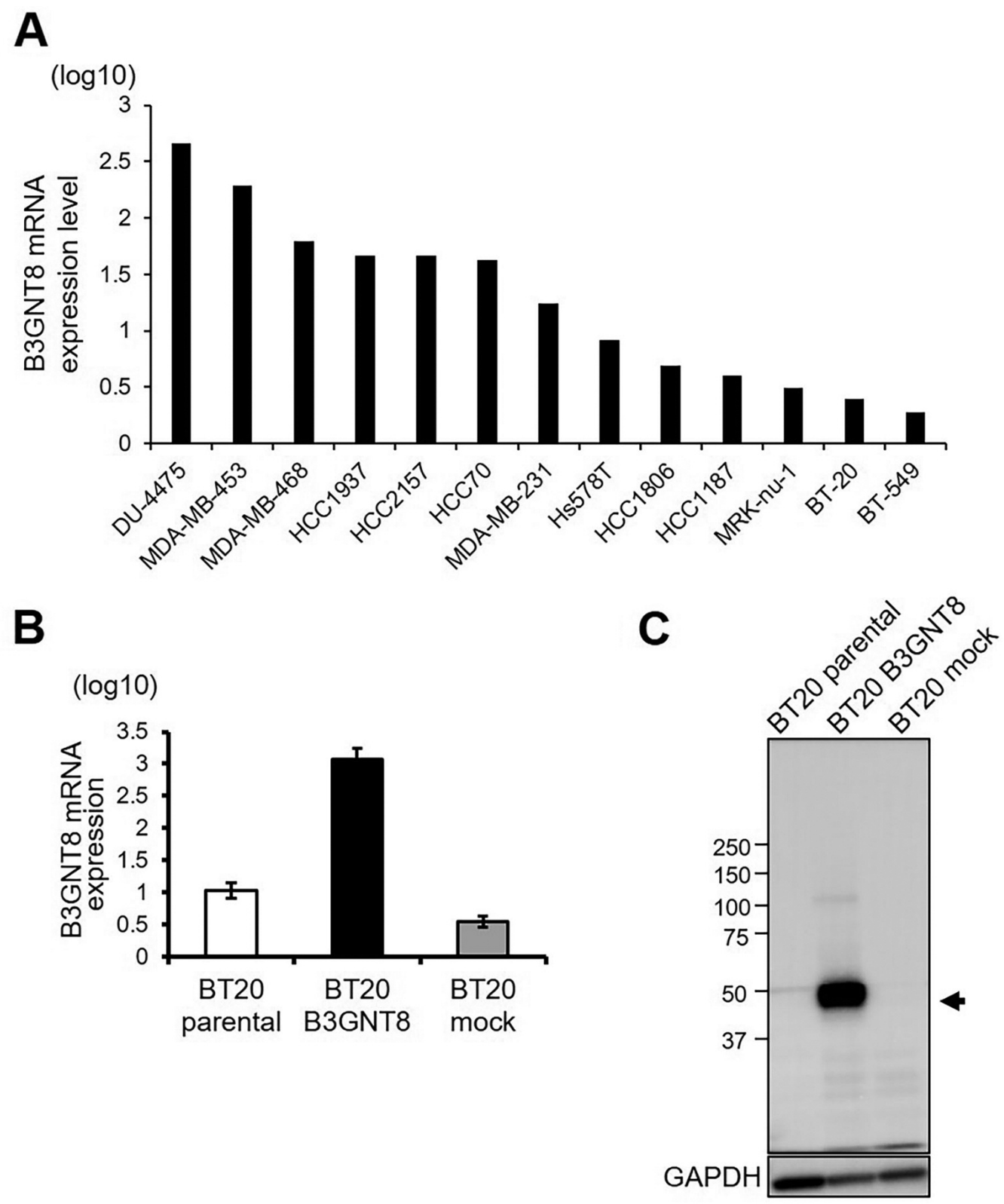

Figure 6. B3GNT8 mRNA and protein expression in BT20 transfectants. (A) B3GNT8 mRNA expression levels were measured in 13 triple-negative breast cancer (TNBC) cell lines by quantitative real-time PCR. Basal-like 1-type TNBC cell lines, as identified by (20), namely MDA-MB-468, HCC1937, and HCC2157, showed relatively high B3GNT8 mRNA levels. (B) B3GNT8 mRNA expression was investigated by quantitative real-time PCR using cDNA prepared from BT20 parental, BT20 B3GNT8-transfected, and BT20 mock cells. Relative B3GNT8 mRNA expression levels normalised to beta-actin are shown. Data shown are from a quantitative real-time PCR conducted in triplicate. (C) B3GNT8 protein expression was investigated by western blotting analysis using a polyclonal anti-B3GNT8 antibody. The arrowhead indicates the expected migration distance for B3GNT8 protein. Twenty microgram of lysate protein were loaded per lane. GAPDH was used as a loading control.

colorectal cancer cells by altering poly- $N$-acetyllactosamine sugars of CD147 (13). In gastric cancer, B3GNT8 expression was found to be regulated by c-jun and the levels shown by immunohistochemistry were high in advanced stages in the TNM classification (23). However, B3GNT8 involvement in breast cancer has not been previously reported.
The current data showed that B3GNT8 mRNA expression levels are associated with relapse and survival status, and that they do not correlate with age, menopausal status, histological subtype, or stage of disease. It has been reported that the pathological complete response rate after anthracycline followed by taxane-based chemotherapy regimens is high in 
patients with BL1 subtype of Lehmann's classification of TNBC $(20,24)$. Thus, the relationship between B3GNT8 expression levels and Lehmann's subtypes were carefully assessed in the present study. B3GNT8 mRNA levels in the BL1 subtype were significantly lower than in other subtypes (Figure 4E). Patients with BL1 subtype respond better to chemotherapy and have a more favourable prognosis compared to patients with other Lehmann TNBCtype-4 subtypes (20). Regarding the relationship between B3GNT8 mRNA expression levels and patient outcomes, higher B3GNT8 mRNA expression was observed in patients who developed distant metastasis within all subtypes (Figure 5C), although significant differences were observed only in BL2 subtype. Taken together, these findings suggest that B3GNT8 might be useful as a prognostic factor regardless of Lehmann's classification. The mechanism through which B3GNT8 results in better survival are unknown and might merit further investigations both in basic and clinical studies.

To further explore the correlation between Lehmann's subtypes and B3GNT8 expression, thirteen patient-derived TNBC cell lines were examined for their B3GNT8 expression levels by quantitative real-time PCR. B3GNT8 expression levels were found to vary among the cell lines (Figure 6A), and cells derived from the BL1-subtype (MDA-MB-468, HCC1937, and HCC2157) showed relatively high levels. Thus, though the number of investigated cell lines is limited, differential expression levels as seen with TCGA data may not hold true for cell lines. Among TNBC cell lines, BT-20 cells were used to overexpress B3GNT8 by transfection. As shown in Figure 6B, C and Figure 7A, the protein was synthesized and apparently localized to the Golgi apparatus.

When the distribution of B3GNT8 protein in TNBC tissue was immunohistochemically investigated using an antiB3GNT8 antibody, small dots in the cytoplasm of breast cancer cells were observed as shown in Figure 7I-L. No such dots were observed in BT-20 cells transfected with B3GNT8 cDNA. The number of anti-B3GNT8 antibody-stained dots in TNBC cells varied within a tumour. We hypothesised that the number of dots roughly corresponds to the amount of B3GNT8 protein, because no other structure was stained by the antibody. Therefore, we counted the number of dots per cancer cell in each specimen. The number of dots was significantly greater in tumour tissue sections from nonrelapsed patients than from relapsed patients, which is contrary to the differences seen with B3GNT8 mRNA levels in the TCGA data set. This discrepancy may be because B3GNT8 mRNA levels do not correspond to the levels of B3GNT8 protein. This possibility needs to be clarified in future investigations. A limitation of the present investigation is that the cohort used in our immunohistochemical studies may be too small to represent the entire TNBC population. Further studies, employing a much larger number of patients across all stages, should clearly be conducted to establish a
Table II. Clinicopathological characteristics of 23 TNBC patients investigated by immunohistochemistry with anti-B3GNT8 antibody. The average observation period was 50.1 months.

\begin{tabular}{lc}
\hline & Number of patients (\%) \\
\hline Age (years) & \\
$30-39$ & $4(17.4)$ \\
$40-49$ & $2(8.7)$ \\
$50-59$ & $5(21.7)$ \\
$60-69$ & $6(26.1)$ \\
70 or greater $^{\text {Menopausal status }}$ & $6(26.1)$ \\
Pre-menopausal $^{\mathrm{a}}$ & \\
Post-menopausal & $9(39.1)$ \\
Unknown & $13(56.5)$ \\
TNM stage & $1(4.4)$ \\
IIA & $14(60.9)$ \\
IIB & $9(39.1)$ \\
\hline
\end{tabular}

aDefined as $<12$ months since last menstrual period AND no prior bilateral ovariectomy AND not on oestrogen replacement; bDefined as prior bilateral ovariectomy $\mathrm{OR}>12$ months since last menstrual period with no prior hysterectomy.

conclusive evidence in the future. Nevertheless, the unique distribution of B3GNT8 protein shown by the binding of anti-B3GNT8 antibody in TNBC cells deserves further investigation. Co-localisation with organelle markers will reveal the subcellular localization of B3GNT8 protein, which is still to be investigated.

In conclusion, the present study demonstrated that the B3GNT8 mRNA expression levels in TNBC tumour tissue are potentially useful in distinguishing patients with favourable and poor clinical outcomes. The biological basis for the prognostic significance of B3GNT8 mRNA expression in TNBC remains the subject of future investigations.

\section{Conflicts of Interest}

All Authors have no potential conflicts of interest to disclose in relation to this study.

\section{Authors' Contributions}

Misato Okazaki conducted all aspects of this study, including the data analysis, studies with cultured cell lines and immunohistochemical analysis. Kaoru Mogushi assisted in data mining and statistical analyses. Haruhiko Fujihira, Miki Noji, and Katrin Ishii-Schrade performed studies with cultured cells and were also engaged in part with immunohistochemical and cytochemical studies. Haruhiko Fujihira, Katrin Ishii-Schrade and Kaori DendaNagai were involved in writing the manuscript. Madoka SatakaMatsuzawa, Katsuya Nakai, and Yoshiya Horimoto selected pathological specimens and provided clinical information associated with the specimens. Mitsue Saito and Tatsuro Irimura designed the study and guided the manuscript preparation. 


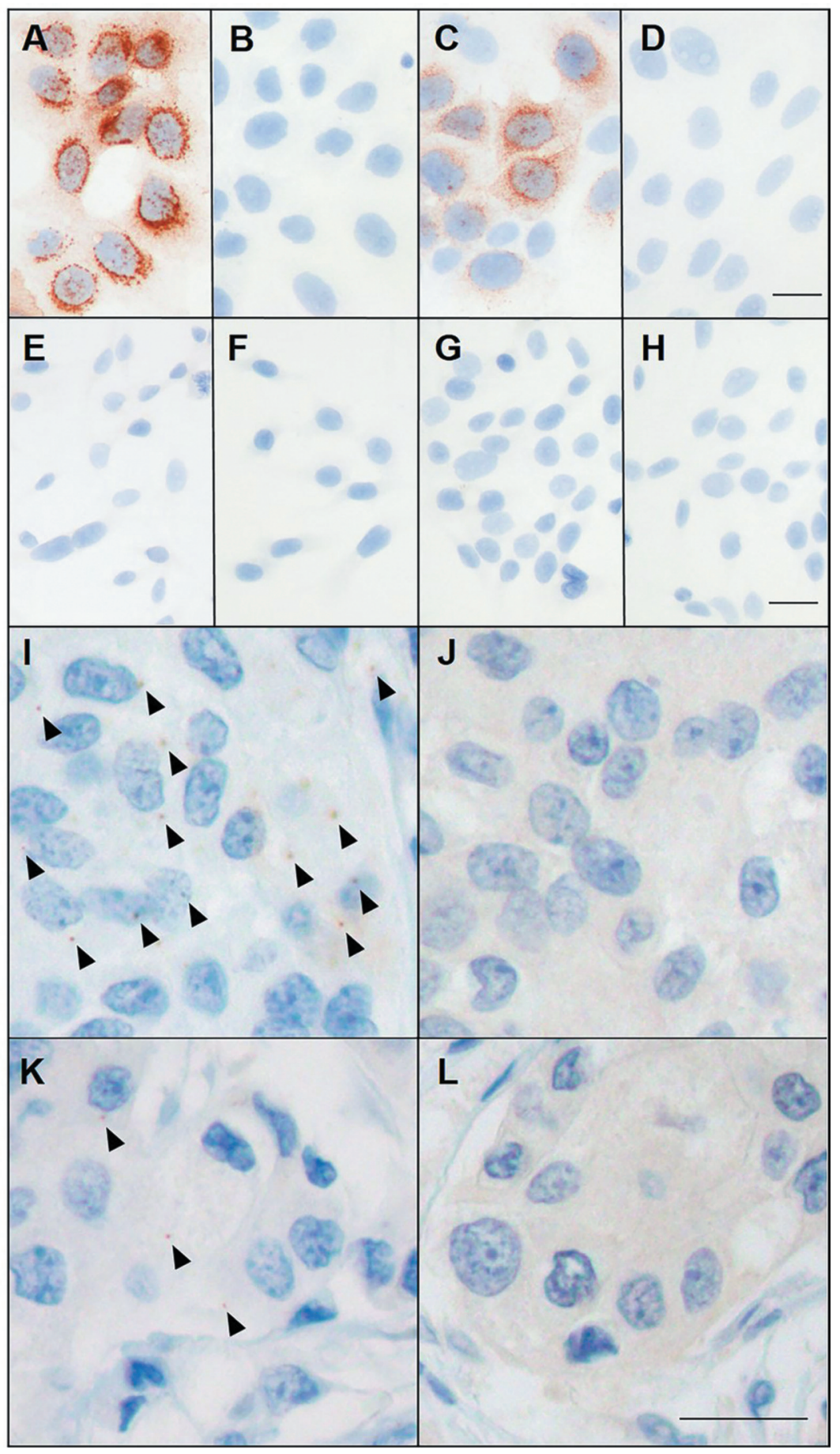

Figure 7. B3GNT8 protein expression in BT-20 cells transfected with B3GNT8-encoding plasmid DNA and in surgical specimens from Stage IIA and IIB TNBC patients. Cells were seeded in chamber slides and paraformaldehyde-fixed before staining. (A) Anti-B3GNT8 polyclonal antibodystained BT-20 cells transfected with B3GNT8, (B) B3GNT8 control antibody-stained BT-20 cells transfected with B3GNT8. (C) Anti-FLAG monoclonal antibody-stained BT-20 cells transfected with B3GNT8. (D) Anti-FLAG isotype control antibody-stained BT-20 cells transfected with B3GNT8. (E) Anti-B3GNT8 polyclonal antibody-stained BT-20 mock cells, (F) B3GNT8 control antibody-stained BT-20 mock cells. (G) Anti-FLAG monoclonal antibody-stained BT-20 mock cells. (H) Anti-FLAG isotype control antibody-stained BT-20 mock cells. (I-L) Representative images from immunohistochemistry staining of 23 surgical specimens with B3GNT8 antibody. Staining of breast cancer tissue was performed on 4- $\mu m$ sections of formalin-fixed and paraffin-embedded surgical specimens. B3GNT8 staining is represented by small dots in the cytoplasm of cancer cells. (I) Anti-B3GNT8 antibody-stained specimen showing many small dots. $(K)$ Anti-B3GNT8 antibody-stained specimen showing few small dots. $(J, L)$ Control antibody-stained surgical specimens. Scale bar shows $25 \mu \mathrm{m}$. Arrowheads indicate the location of dot-like staining. 
A

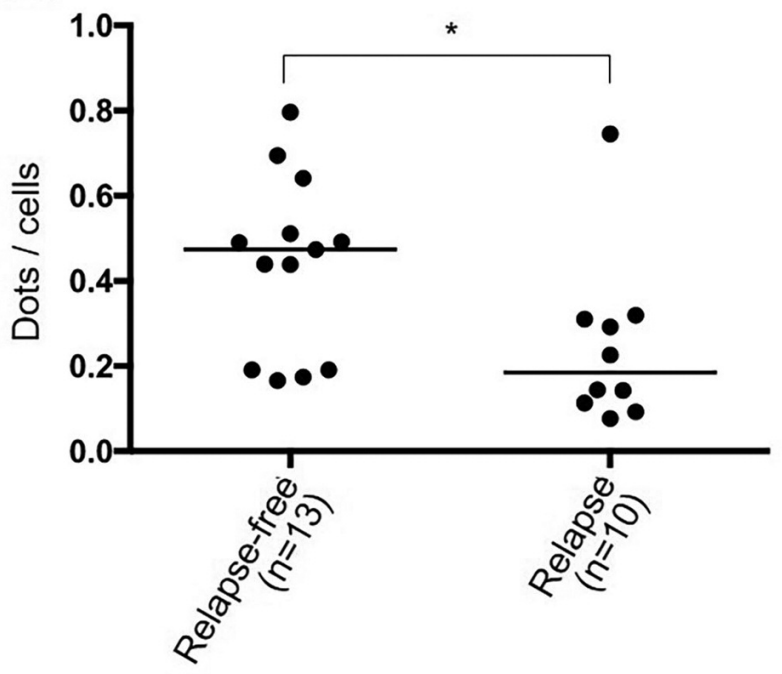

C
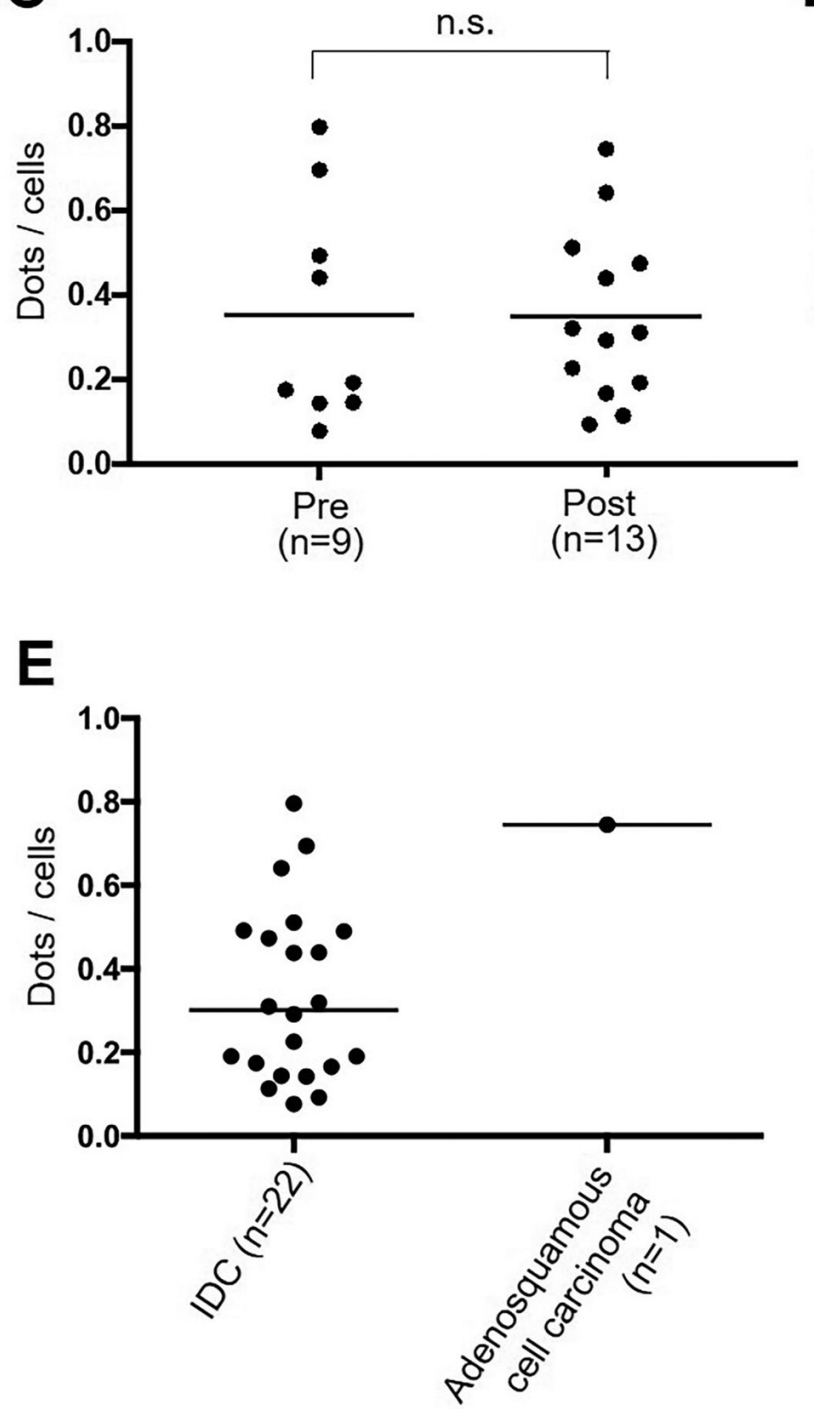

B

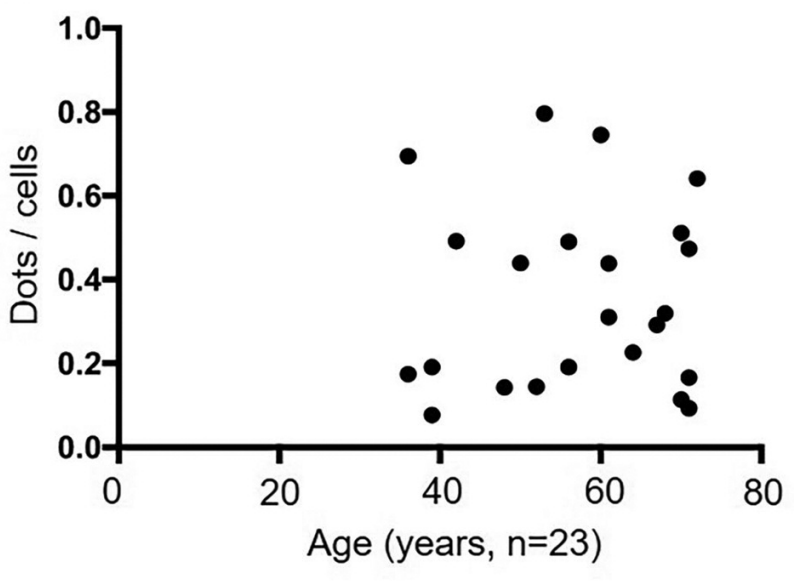

D

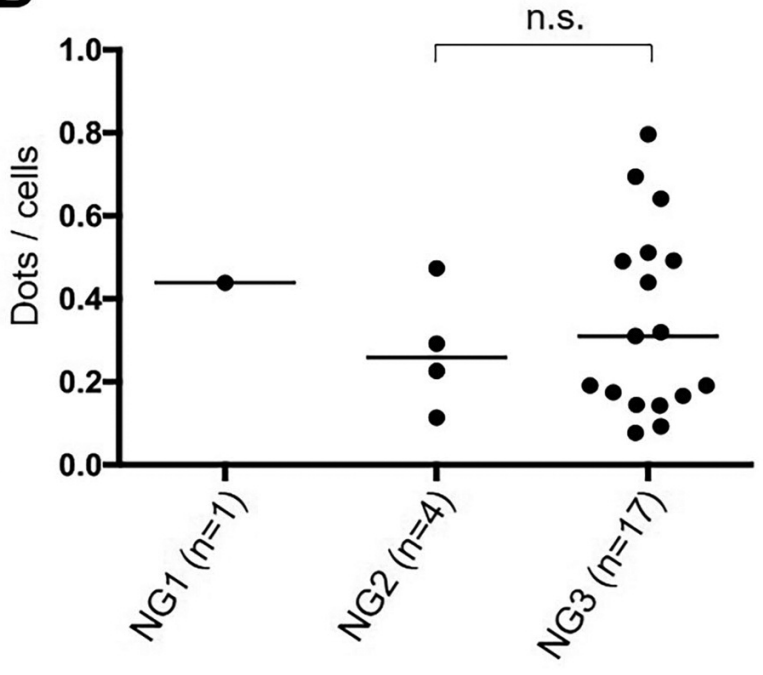

Figure 8. Relationship of B3GNT8 protein level with clinicopathological variables in 23 TNBC tumour specimens as investigated by immunohistochemistry using a polyclonal anti-B3GNT8 antibody. B3GNT8 staining was observed as small dot-like structures in the cytoplasm of TNBC cells. The dot-like structures were assumed to represent B3GNT8 protein. Three locations in each specimen were chosen and dots representing B3GNT8 protein were counted versus the number of cancer cells in a specified counting area (91,000 um2). Each dot in the figure represents the dot per cell count for one individual patient. (A) B3GNT8 staining and recurrence-free survival (RFS) status. (B) B3GNT8 staining and age. (C) B3GNT8 staining and menopausal status. One patient's menopausal status was not classifiable and is not shown. (D) B3GNT8 staining and nuclear grade (one patient's nuclear grade was not available, and this patient is not shown). (E) B3GNT8 staining and histological diagnosis. Mann-Whitney U-test. n.s.: not significant, ${ }^{*} p<0.05$. The median is shown as a horizontal line. $N G$ : Nuclear grade; IDC: invasive ductal carcinoma. 


\section{Acknowledgements}

This work was supported as part of the AMED Project by the Ministry of Economy, Trade and Industry (METI) (JP19ae0101026). The Authors wish to thank Drs. Takashi Sato and Kiyohiko Angata (National Institute of Advanced Industrial Science and Technology) for kindly providing B3GNT8 expression vectors. Further, the Authors would like to thank Dr. Yasunori Chiba (National Institute of Advanced Industrial Science and Technology) for valuable comments. The Authors also like to thank the members of the Laboratory of Morphology and Image Analysis, the Division of Cell Biology, and the Laboratory of Molecular and Biochemical Research, Research Support Center, Juntendo University Graduate School of Medicine, for technical assistance.

\section{References}

1 Cocco S, Piezzo M, Calabrese A, Cianniello D, Caputo R, Lauro VD, Fusco G, Gioia GD, Licenziato $M$ and Laurentiis M: Biomarkers in triple-negative breast cancer: State-of-the-art and future perspectives. Int J Mol Sci 21(13): 4579, 2020. PMID: 32605126. DOI: $10.3390 /$ ijms 21134579

2 Dent R, Trudeau M, Pritchard KI, Hanna WM, Kahn HK, Sawka CA, Lickley LA, Rawlinson E, Sun P and Narod SA: Triplenegative breast cancer: Clinical features and patterns of recurrence. Clin Cancer Res 13(15 Pt 1): 4429-4434, 2007. PMID: 17671126. DOI: 10.1158/1078-0432.Ccr-06-3045

3 Symmans WF, Peintinger F, Hatzis C, Rajan R, Kuerer H, Valero V, Assad L, Poniecka A, Hennessy B, Green M, Buzdar AU, Singletary SE, Hortobagyi GN and Pusztai L: Measurement of residual breast cancer burden to predict survival after neoadjuvant chemotherapy. J Clin Oncol 25(28): 4414-4422, 2007. PMID: 17785706 . DOI: $10.1200 /$ jco.2007.10.6823

4 Costa AF, Campos D, Reis CA and Gomes C: Targeting glycosylation: A new road for cancer drug discovery. Trends Cancer 6(9): 757-766, 2020. PMID: 32381431. DOI: 10.1016/j.trecan.2020.04.002

5 Pinho SS and Reis CA: Glycosylation in cancer: Mechanisms and clinical implications. Nat Rev Cancer 15(9): 540-555, 2015. PMID: 26289314. DOI: $10.1038 / \mathrm{nrc} 3982$

6 Burchell JM, Beatson R, Graham R, Taylor-Papadimitriou J and Tajadura-Ortega V: O-linked mucin-type glycosylation in breast cancer. Biochem Soc Trans 46(4): 779-788, 2018. PMID: 29903935. DOI: 10.1042/BST20170483

7 Song KH, Park MS, Nandu TS, Gadad S, Kim SC and Kim MY: Galnt14 promotes lung-specific breast cancer metastasis by modulating self-renewal and interaction with the lung microenvironment. Nat Commun 7: 13796, 2016. PMID: 27982029. DOI: $10.1038 /$ ncomms 13796

8 Fang R, Xu F, Shi H, Wu Y, Cao C, Li H, Ye K, Zhang Y, Liu $\mathrm{Q}$, Zhang S, Zhang $\mathrm{W}$ and Ye L: Lamtor5 raises abnormal initiation of o-glycosylation in breast cancer metastasis via modulating galnt1 activity. Oncogene 39(11): 2290-2304, 2020. PMID: 31836847. DOI: 10.1038/s41388-019-1146-2

9 Seko A and Yamashita K: Activation of beta1,3-nacetylglucosaminyltransferase-2 (beta3gn-t2) by beta3gn-t8. Possible involvement of beta3gn-t8 in increasing poly-nacetyllactosamine chains in differentiated hl-60 cells. J Biol Chem 283(48): 33094-33100, 2008. PMID: 18826941. DOI: 10.1074/jbc.M806933200
10 Togayachi A, Kozono Y, Kuno A, Ohkura T, Sato T, Hirabayashi J, Ikehara Y and Narimatsu H: Beta3gnt2 (b3gnt2), a major polylactosamine synthase: Analysis of b3gnt2-deficient mice. Methods Enzymol 479: 185-204, 2010. PMID: 20816167. DOI: 10.1016/s0076-6879(10)79011-x

11 Ishida H, Togayachi A, Sakai T, Iwai T, Hiruma T, Sato T, Okubo R, Inaba N, Kudo T, Gotoh M, Shoda J, Tanaka N and Narimatsu H: A novel beta1,3-n-acetylglucosaminyltransferase (beta3gn-t8), which synthesizes poly-n-acetyllactosamine, is dramatically upregulated in colon cancer. FEBS Lett 579(1): 7178, 2005. PMID: 15620693. DOI: 10.1016/j.febslet.2004.11.037

12 Dennis JW, Granovsky M and Warren CE: Glycoprotein glycosylation and cancer progression. Biochim Biophys Acta 1473(1): 21-34, 1999. PMID: 10580127. DOI: 10.1016/s03044165(99)00167-1

13 Ni J, Jiang Z, Shen L, Gao L, Yu M, Xu X, Zou S, Hua D and Wu S: Beta3gnt8 regulates the metastatic potential of colorectal carcinoma cells by altering the glycosylation of cd147. Oncol Rep 31(4): 1795-1801, 2014. PMID: 24573103. DOI: 10.3892/or.2014. 3042

14 Jiang Z, Zhang H, Liu C, Yin J, Tong S, Lv J, Wei S and Wu S: Beta3gnt8 promotes colorectal cancer cells invasion via cd147/mmp2/galectin3 axis. Front Physiol 9: 588, 2018. PMID: 29875690. DOI: 10.3389/fphys.2018.00588

15 Shen L, Yu M, Xu X, Gao L, Ni J, Luo Z and Wu S: Knockdown of beta3gnt8 reverses 5 -fluorouracil resistance in human colorectal cancer cells via inhibition the biosynthesis of polylactosamine-type n-glycans. Int J Oncol 45(6): 2560-2568, 2014. PMID: 25269761. DOI: 10.3892/ijo.2014.2672

16 cBioPortal For Cancer Genomics: Data set: Data_rna_ seq_v2_expression_median.Txt. Available at: http://www. cbioportal.org/ [Last accessed on December 29, 2019]

17 Weinstein JN, Collisson EA, Mills GB, Shaw KR, Ozenberger BA, Ellrott K, Shmulevich I, Sander C and Stuart JM: The cancer genome atlas pan-cancer analysis project. Nat Genet 45(10): 11131120, 2013. PMID: 24071849. DOI: 10.1038/ng.2764

18 The R Foundation: The $\mathrm{r}$ project for statistical computing. Available at: https://www.r-project.org/ [Last accessed on December 29, 2019]

19 Sorlie T, Perou CM, Tibshirani R, Aas T, Geisler S, Johnsen H, Hastie T, Eisen MB, van de Rijn M, Jeffrey SS, Thorsen T, Quist $\mathrm{H}$, Matese JC, Brown PO, Botstein D, Lonning PE and Borresen-Dale AL: Gene expression patterns of breast carcinomas distinguish tumor subclasses with clinical implications. Proc Natl Acad Sci USA 98(19): 10869-10874, 2001. PMID: 11553815. DOI: 10.1073/pnas.191367098

20 Lehmann BD, Jovanovic B, Chen X, Estrada MV, Johnson KN, Shyr Y, Moses HL, Sanders ME and Pietenpol JA: Refinement of triple-negative breast cancer molecular subtypes: Implications for neoadjuvant chemotherapy selection. PLoS One 11(6): e0157368, 2016. PMID: 27310713. DOI: 10.1371/journal.pone.0157368

21 Gyorffy B, Lanczky A, Eklund AC, Denkert C, Budczies J, Li Q and Szallasi Z: An online survival analysis tool to rapidly assess the effect of 22,277 genes on breast cancer prognosis using microarray data of 1,809 patients. Breast Cancer Res Treat 123(3): 725-731, 2010. PMID: 20020197. DOI: 10.1007/s10549009-0674-9

22 Mihaly Z, Kormos M, Lanczky A, Dank M, Budczies J, Szasz MA and Gyorffy B: A meta-analysis of gene expression-based biomarkers predicting outcome after tamoxifen treatment in 
breast cancer. Breast Cancer Res Treat 140(2): 219-232, 2013. PMID: 23836010. DOI: 10.1007/s10549-013-2622-y

23 Jiang Z, Liu Z, Zou S, Ni J, Shen L, Zhou Y, Hua D and Wu S: Transcription factor c-jun regulates beta3gn-t8 expression in gastric cancer cell line sgc-7901. Oncol Rep 36(3): 1353-1360, 2016. PMID: 27459970. DOI: 10.3892/or.2016.4959

24 Masuda H, Baggerly KA, Wang Y, Zhang Y, Gonzalez-Angulo AM, Meric-Bernstam F, Valero V, Lehmann BD, Pietenpol JA, Hortobagyi GN, Symmans WF and Ueno NT: Differential response to neoadjuvant chemotherapy among 7 triple-negative breast cancer molecular subtypes. Clin Cancer Res 19(19): 5533-5540, 2013. PMID: 23948975. DOI: 10.1158/1078-0432.CCR-13-0799

25 Kaplan Meier Plotter: Breast cancer. Available at: http:// kmplot.com/analysis/index.php? $\mathrm{p}=$ service \& cancer=breast [Last accessed on January 4, 2021]

Received December 15, 2020

Revised January 6, 2021

Accepted January 7, 2021 